\title{
CONDUCTIVIDAD Y SALINIDAD EN LOS ECOSISTEMAS ACUATICOS DEL PARQUE NACIONAL DE DOÑANA (SO, ESPAÑA)
}

\author{
O. G. Bodelón, M. Bernués, A. Baltanás \& $\boldsymbol{C}$. Montes \\ Departamento Interuniversitario de Ecología, Universidad Autónoma de Madrid, 28049-Madrid, España.
}

Palabras clave: Bajo Guadalquivir, Doñana, marisma, ecosistemas acuáticos sobre manto eólico, concentración iónica, clorosidad.

\author{
ABSTRACT \\ CONDUCTIVITY AND SALINITY IN AQUATIC ECOSYSTEMS OF DOÑANA NATIONAL PARK (SW, SPAIN)
}

(5)

A large data set of chemical valiables from an extensive survey of the water bodies of Doñana has been used for assessing the relationship between conductivity and salinity in the area. The aim of finding an appropriate conversion factor for estimating salinity from conductivity data has rendered a better knowledge of factors affecting that relationship. A table is offered for computing in an easy way predicted salinities. However, and despite the optimum fit of data to the model, estimations remain loose when dealing with highly mineralized waters. The relationship between clorosity and salinity has been also evaluated and discarded as useful due to important flaws in regression model assumptions.

\section{INTRODUCCIÓN}

Los ecosistemas acuáticos constituyen uno de los elementos más característicos que definen el paisaje del Parque Nacional de Doñana. Desde una perspectiva genético-funcional el Parque se compone de cuatro sistemas ambientales naturales (continental, eólico, marisma y costero) que mantienen un rico y variado patrimonio de formaciones palustres y criptohumedales de carácter hipo- y epigénico (BRAVO \& MONTES, 1993). En todos estos ecosistemas se distinguen una serie de factores claves que condicionan los procesos que definen su integridad ecológica a una escala espacial amplia. Entre estos factores (régimen hidrológico, turbiedad y estado trófico) la salinidad constituye uno de los descriptores básicos de la tipología funcional de los humedales del Parque (BERNUES, 1990).

En este sentido, disponer de un método fiable y rápido de estimación directa de la salinidad es de una gran importancia tanto para el desarrollo de estudios básicos como para la realización de programas de seguimiento y gestión ambiental (WILLIAMS, 1986). Dado que los métodos habitualmente utilizados para la medida directa de la salinidad (concentración de iones mayoritarios y residuo seco a $105^{\circ}$-TDS-) suponen un esfuerzo notable tanto en tiempo como en dinero, se han utilizado frecuentemente métodos indirectos alternativos entre los que destaca la conductividad eléctrica (HEURTEAUX, 1988).
Disponer de un factor de conversión que relacione la conductividad con la salinidad constituye, por lo tanto, una herramienta de trabajo de gran utilidad (HAMMER, 1986). Para aguas salobres (de origen marino) en las que los cloruros representan alrededor del $50 \%$ del peso total de sales disueltas, se emplea también la clorosidad (concentración del ion cloruro expresada como peso por unidad de volumen) para estimar la salinidad a través de la fórmula de KUNDSEN (1902): $\mathbf{S} \% o=0.03+1.805 \mathrm{Cl}\left(\mathrm{gl}^{-1}\right)$.

El uso de la conductividad como estimador de la salinidad plantea problemas en distritos limnológicos hidroquímicamente muy heterogéneos en su concentración y composición iónica (WILLIAMS, 1986; FLORÍN et al., 1993; ALCORLO et al., en prep.). En el Parque Nacional de Doñana, sin embargo, la presencia de aguas tanto superficiales (BERNUES, 1990) como subterráneas (MANZANO et al., 1991) de características homogéneas en su composición iónica, con predominio de aguas clorurado-sódicas y escasas singularidades de carácter bicarbonatado-cálcico, justifica el empleo de esta aproximación metodológica.

\section{MÉTODOS}

Se estudiaron 391 muestras de agua procedentes de 199 localidades de muestreo repartidas por todo el territorio del Parque Nacional e incluyendo los distintos tipos funcionales de

Limnética, 10 (2): $27-31$ (1994)

O Asociación Española de Limnología, Madrid. Spain 
ecosistemas acuáticos representados en la zona. Las muestras se recogieron entre los años 1988 y 1993 en distintas fases de su ciclo anual con la idea de abarcar el máximo rango posible de variación en la concentración iónica de sus aguas. La conductividad se midió con un conductivímetro modelo INSTRAN 10 con compensación automática de temperatura (cte. de célula=1). La salinidad se estimó como la suma de las concentraciones de los componentes iónicos mayoritarios $\left(\mathrm{Ca}^{++}\right.$, $\mathrm{Mg}^{++}, \mathrm{Na}^{+}, \mathrm{K}^{+}, \mathrm{CO}_{3}=, \mathrm{CO}_{3} \mathrm{H}^{-}, \mathrm{SO}_{4}=\mathrm{Cl}^{-}$) medidos según la metodología recomendada en APHA $(1985,1989)$.

La relación salinidad-conductividad se evaluó mediante análisis de regresión lineal (mínimos cuadrados) sobre los datos transformados logarítmicamente. El empleo del modelo de regresión lineal sobre datos logaritmizados se justifica por corregir la heterocedasticidad de los mismos (WILLIAMS, 1986) y normalizar la distribución de los residuos. Es importante destacar que el uso de variables transformadas requiere, para la aplicación del modelo a casos reales (predicciones futuras), una transformación inversa que permita obtener estimas de la variable dependiente y sus bandas de confianza en una escala aritmética y no logarítmica. Aunque con frecuencia se asume que esta transformación, en el caso del modelo logtransformado que nos ocupa, es el antilogaritmo del valor correspondiente, este procedimiento es erróneo produciendo sesgos en las estimaciones que pueden ser importantes. Una alternativa adecuada es la propuesta por BASKERVILLE (1976)

$$
S=\operatorname{anti} \log \left(\log (\hat{S})+\mathbf{s}^{2} \log (\hat{S} / 2)\right.
$$

donde $S$ representa a los valores de la salinidad en escala aritmética (ver ALCORLO et al., in prep. para un tratamiento más amplio de esta cuestión).

Para estimar la correción de los resultados analíticos se calculó el balance iónico con la siguiente expresión

$\%$ error=100( $\sum$ cationes- $\sum$ aniones $) /\left(\sum\right.$ cationes $+\sum$ aniones $)($ APHA,1989) desechándose aquellas muestras que presentaban un error superior al $10 \%$, de forma que el conjunto final de datos quedó reducido a 358 muestras.

Dado que el objetivo principal es obtener una herramienta predictiva para la estimación de la salinidad a partir de las medidas de conductividad, es necesario obtener una idea de la exactitud de dicha estima. Para ello se calculó la banda de confianza asociada a observaciones futuras unitarias usando la expresión de ZAR (1984, p.275).

De forma similar, y dado su empleo generalizado en algunos ambientes salobres, se estudió la relación entre salinidad y clorosidad mediante análisis de regresión lineal. En esta ocasión, sin embargo, los datos no se transformaron puesto que la expresión de KNUDSEN (1902) se refiere a datos brutos.

\section{RESULTADOS Y DISCUSIÓN}

El análisis de la relación entre salinidad y conductividad resulta en el siguiente modelo lineal (Fig. 1),

$\log _{10}($ Sal $)=-0.175+1.0053 \log _{10}($ Cond $)(n=358) r^{2}=0.99$ que expresa la relación entre conductividad y salinidad para el área considerada.

Tanto la constante como la pendiente de esta regresión son significativamente diferentes de $0(\mathrm{p}<0.0001)$ y el modelo en su conjunto es altamente significativo $(F=38534.62$, $\mathrm{p}<0.0001$ ). La pendiente del modelo, sin embargo, no es significativamente distinta de $\mathbf{1}$ (error estándar asociado a la estima de la pendiente igual a 0.005 ) coincidiendo con otros modelos que, desarrollados también para aguas clorurado-sódicas (ej. WILLIAMS, 1986). muestran una pendiente muy similar.

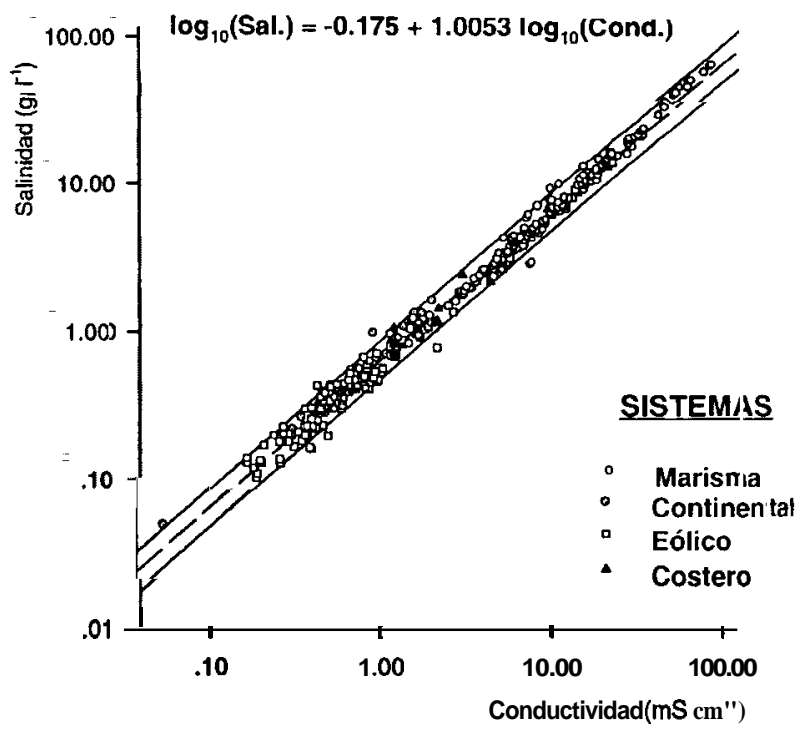

FIGURA 1. Modelo de regresión lineal para la relación salinidad-conductividad en masas de agua del Parque Nacional de Doñana (los diferentes símbolos indican los diferentes sectores ambientales). Las líneas continuas marcan las bandas de confianza $(95 \%)$ de la predicción de una observación futura.

FIGURE 1. Lineal regression model of the relationshipbetween salinity and conductivity in water bodies of Doñana National Park (different areas are depicted with different symbols). Solid lines are the confidence bands $(95 \%)$ for the future predictions.

La figura 1 muestra también las bandas de confianza (95\%) de la predicción para el conjunto de las muestras. Considerando que el objetivo principal de derivar una relación entre conductividad y salinidad es obtener una herramienta eficaz de estima de la segunda a partir de la primera, se ha confeccionado una tabla que permite hacer predicciones de forma rápida (Tabla l). En esta tabla se puede apreciar que, para conductivi. 
dades elevadas, las predicciones tienden a hacerse poco precisas (ej., para una conductividad observada de $90 \mathrm{mS} \mathrm{cm}^{-1}$ la banda de predicción de la salinidad tiene una anchura de, aproximadamente, $38 \mathrm{~g}^{\mathbf{l}^{-1}}$ ). El efecto de aumento de la anchura de la banda de confianza de las predicciones conforme nos movemos hacia valores elevados de conductividad no se aprecia en la relación de la Fig. 1 puesto que, al haberse realizado en escala logarítmica, las bandas de confianza aparecen con anchura constante, casi paralelas. Sin embargo, la misma información dibujada en escala aritmética presentaría las bandas de confianza en expansión que refleja la Tabla 1. Este patrón no hace sino manifestar la incorporación al modelo de la naturaleza típicamente heterocedástica de los datos que tienen una mayor varianza conforme nos movemos a las zonas de mineralización más elevada.
Si bien a elevadas concentraciones iónicas las interferencias, los equilibrios químicos inestables y los efectos dieléctricos y electrostáticos se traducen en una mayor variabilidad de la relación conductividad-salinidad (WILLIAMS, 1966) no es menos cierto que una parte de la misma sea posiblemente de carácter instrumental y pueda corregirse mediante el empleo de los conductímetros de células de constante adecuada a la salinidad del medio (M ${ }^{\circ}$ AGRICULTURA, 1986, BERNUÉS, 1990). Es también posible que un modelo como el aquí obtenido deba sus imprecisiones a haber combinado en un sólo conjunto datos procedentes de unidades ambientales con comportamientos bien diferentes en su relación conductividad salinidad. Analizados por separado las muestras de cada uno de los sistemas ambientales de Doñana, los resultados muestran, sin embargo, comportamientos similares en todos ellos para la

TABLA 1 Bandas de confianza (95\%) estimadas para la salinidad $\left(\mathrm{g}^{1}\right)$ a partir de valores de conductividad en el intervalo de 1 a $100 \mathrm{mS} \mathrm{cm}^{1}$ TABLE 1 Estimated confidence bands $(95 \%)$ of salinity $\left(\mathrm{g}^{1}\right)$ computed from conductivity values ranging from 1 to $100 \mathrm{mS} \mathrm{cm}{ }^{1}$

\begin{tabular}{|c|c|c|c|c|c|}
\hline $\begin{array}{c}\text { Cond. } \\
(\mathrm{mS} \mathrm{cm-')}\end{array}$ & $\begin{array}{l}\text { Salinidad } \\
\qquad\left(\mathbf{g ~ l}^{-1}\right) \\
\text { L.C. }(95 \%)\end{array}$ & $\begin{array}{c}\text { Cond. } \\
\left(\mathrm{mS} \mathrm{cm}^{-}\right)\end{array}$ & $\begin{array}{l}\text { Salinidad } \\
\quad\left(\mathbf{g ~ l}^{\mathbf{1}}\right) \\
\text { L.C. }(95 \%)\end{array}$ & $\begin{array}{c}\text { Cond. } \\
(\mathbf{m S ~ c m - ' )}\end{array}$ & $\begin{array}{l}\text { Salinidad } \\
\quad\left(\mathrm{g} \mathrm{l}^{-1}\right) \\
\text { L.C. }(95 \%)\end{array}$ \\
\hline 1.00 & $0.50-0.91$ & 26.00 & $13.28-24.08$ & 52.00 & $26.64-48.37$ \\
\hline 2.00 & $1.01-1.83$ & 27.00 & $13.79-25.01$ & 54.00 & $27.67-50.24$ \\
\hline 3.00 & $1.52-2.74$ & 28.00 & $14.31-25.94$ & 56.00 & $28.70-52.11$ \\
\hline 4.00 & $2.02-3.66$ & 29.00 & $14.82-26.87$ & 58.00 & $29.73-53.98$ \\
\hline 5.00 & $2.53-4.59$ & 30.00 & $15.33-27.81$ & 60.00 & $30.76-55.86$ \\
\hline 6.00 & $3.04-5.51$ & 31.00 & $15.85-28.74$ & 62.00 & $31.79-57.73$ \\
\hline 7.00 & $3.55-6.43$ & 32.00 & $16.36-29.67$ & 64.00 & $32.82-59.61$ \\
\hline 8.00 & $4.06-7.36$ & 33.00 & $16.87-30.61$ & 66.00 & $33.85-61.48$ \\
\hline 9.00 & $4.57-8.28$ & 34.00 & $17.39-31.54$ & 68.00 & $34.88-63.36$ \\
\hline 10.00 & $5.08-9.21$ & 35.00 & $17.90-32.47$ & 70.00 & $35.91-65.23$ \\
\hline 11.00 & $5.60-10.13$ & 36.00 & $18.41-33.41$ & 72.00 & $36.94-67.11$ \\
\hline 12.00 & $6.11-11.06$ & 37.00 & $18.93-34.34$ & 74.00 & $37.97-68.99$ \\
\hline 13.00 & $6.62-11.99$ & 38.00 & $19.44-35.27$ & 76.00 & $39.00-70.86$ \\
\hline 14.00 & $7.13-12.92$ & 39.00 & $19.96-36.21$ & 78.00 & $40.03-72.74$ \\
\hline 15.00 & $7.64-13.84$ & 40.00 & $20.47-37.14$ & 80.00 & $41.06-74.62$ \\
\hline 16.00 & $8.15-14.77$ & 41.00 & $20.98-38.08$ & 82.00 & $42.10-76.49$ \\
\hline 17.00 & $8.67-15.70$ & 42.00 & $21.50-39.01$ & 84.00 & $43.13-78.37$ \\
\hline 18.00 & $9.18-16.63$ & 43.00 & $22.01-39.95$ & 86.00 & $44.16-80.25$ \\
\hline 19.00 & $9.69-17.56$ & 44.00 & $22.53-40.88$ & 88.00 & $45.19-82.13$ \\
\hline 20.00 & $10.20-18.49$ & 45.00 & $23.04-41.82$ & 90.00 & $46.22-84.01$ \\
\hline 21.00 & $10.72-19.42$ & 46.00 & $23.56-42.75$ & 92.00 & $47.25-85.89$ \\
\hline 22.00 & $11.23-20.35$ & 47.00 & $24.07-43.69$ & 94.00 & $48.28-87.77$ \\
\hline 23.00 & $11.74-21.28$ & 48.00 & $24.58-44.62$ & 96.00 & $49.32-89.65$ \\
\hline 24.00 & $12.25-22.21$ & 49.00 & $25.10-45.56$ & 98.00 & $50.35-91.53$ \\
\hline 25.00 & $12.77-23.15$ & 50.00 & $25.61-46.49$ & 100.00 & $51.38-93.41$ \\
\hline
\end{tabular}


mencionada relación (Tabla 2). Existe, no obstante, una diferencia pequeña pero estadísticamente significativa $\left(\mathrm{F}_{\mathrm{obs} .3 .352}=\right.$ $3.955, \mathrm{p}<0.05$ ) en el comportamiento del sector de marisma frente a los restantes. El hecho de que este sector sea el más abundantemente representado en el conjunto de datos y que a él pertenezcan las láminas de agua con salinidades más elevadas no permite, por el momento, interpretar con certeza esta diferencia, que puede responder tanto a una realidad objetiva como a un artefacto estadístico.

TABLA 2. Valores del modelo potencial (datos log-transformados) obtenidos para cada uno de los sistemas ambientales del Parque Nacional de Doñana. TABLE 2. Regression analysis coefficients (log-transformed data) for each of the environmental systems in Doñana National Park.

\begin{tabular}{lrccc}
\hline SECTOR & \multicolumn{1}{c}{$\mathrm{n}$} & Constante & Pendiente & $\mathrm{r}^{2}$ \\
\hline Marisma & 157 & -0.655 & 1.032 & 0.986 \\
Continental & 29 & -0.137 & 0.969 & 0.966 \\
Eólico & 149 & -0.328 & 0.984 & 0.983 \\
Costero & 18 & -0.219 & 0.979 & 0.978 \\
\hline
\end{tabular}

Especial atención merece la estimación de la salinidad a partir de la clorosidad (concentración de iones cloruro en volumen de disolución).Este método ha sido tradicionalmenteempleado en el campo de la oceanografía y, por extensión, en ambientes continentales de carácter talásico (eg. Camarga) y se basa en la conocida relación de KNUDSEN (1902): $\mathbf{S} \%$ o $0.03+1.805$ $\left(\mathrm{Cl}^{-}\right)$. Estimada esta relación para las aguas del Parque Nacional de Doñana el modelo de regresión obtenido es

$$
\mathrm{S} \%_{0}=0.24+1.732\left(\mathrm{Cl}^{-}\right) \quad \mathrm{r}^{2}=0.99, \mathrm{n}=364
$$

expresión muy similar a la propuesta por KNUDSEN (1902). Un análisis detallado de los residuos del análisis de regresión pone de manifiesto, sin embargo, los problemas asociados a esta aproximación metodológica. Como queda patente en la Fig. 2 la distribución de los residuos adolece de una notable no-normalidad, lo que provoca inestabilidad en los errores asociados a las estimas de los coeficientes y hace cuando menos sospechosas las inferencias de ellos derivadas (límites de confianza, predicciones...) Por ello, no recomendamos el uso de esta relación para los ambientes acuáticos de Doñana.

Como conclusión podemos afirmar que el número de muestras analizadas para el conjunto del Parque es lo suficientemente elevado, y recoge adecuadamente su variabilidad espacial y temporal, para que el modelo aquí derivado pueda considerarse como satisfactoriamente representativo. Si bien es cierto que la recogida de más datos puede permitir la mejora del modelo, no creemos que éste varíe de forma sustancial, especialmente en lo que se refiere a las predicciones. Consideramos, por ello,

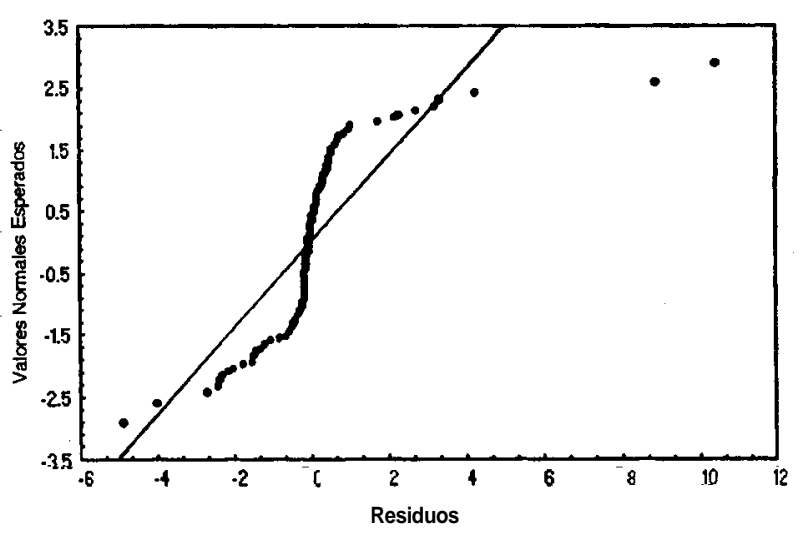

FIGURA 2. Diagrama de probabilidad de los residuos derivados del modelo de regresión lineal salinidad-clorosidad. La línea replesenta los valores esperados para el caso de la distribución normal asumida por el modelo, los puntos son los valores reales obtenidos.

FIGURE 2. Normal probability plot of residuals of the salinity-clorosity regression model. Solid correponds to the expected normal distribution of residuals, points are actual values.

que el modelo general puede aplicarse a todo el temtorio del Parque, independientemente del sector ambiental al que nos estemos refiriendo, especialmente para estudios de carácter extensivo y de gestión. Se recomienda, sin embargo, que en los estudios específicos en los que quiera emplearse la salinidad como una variable del sistema, ésta sea calculada mediante los métodos analíticos al uso y no a través de sus relaciones con otras variables que se demuestran, especialmente en masas de agua de conductividad elevada, como poco adecuadas para realizar predicciones razonables.

\section{AGRADECIMIENTOS}

Este trabajo no hubiera sido posible sin la colaboración que tanto el ICONA como el CSIC (Estación Biológica de Doñana) y los guardas del Parque nos han prestado en la realización de diversos trabajos que han servido de base para la elaboración de este estudio. Nuestro especial agradecimiento a Chans por su constante apoyo, y a la manzanilla por alegrar los momentos difíciles.

\section{BIBLIOGRAFÍA}

AlCorlo, P., BALTANÁs, A. \& C. MONTES, Is it possible to estimate salinity from conductivity data in Ibelian saline lakes? (En preparación).

APHA, 1985, 1989. Standard methods for the examination of water and wastewater. $16^{\text {th }} \& 17^{\text {th }}$ ed.

BASKERVILLE, G.H. 1972. Use of logalithmic regression in 
the estimation of plant biomass. Can. J. Forest. Research 2: 49-53.

BERNUÉS, M. 1990. Limnología de los ecosistemas acuáticos del Parque Nacional de Doñana. Tesis Doctoral, Universidad Autónoma de Madrid.

BRAVO, M.A. \& C. MONTES 1993. Inventario de las formaciones palustres del manto eólico del Parque Nacional de Doñana (SW España). Actas del VI Congreso Español de Limnología, Granada: 31-43.

FLORÍN, M.,; MALTCHIK, L.; MOLLÁ, S.; BALSA, J. \& C. MONTES .1993. Relaciones conductividad-concentración iónica en las aguas de las lagunas salinas españolas. Actas И Congreso Español de Limnología: 83-90.

HAMMER, U.T. 1986. Saline lake ecosystems of the world. Junk Publ. 616 pp.

HEURTEAUX, P. 1988. Mesure de la salinité des eaux naturelles en hydrologie et en hydrobiologie. Essai por une standardisation de I'expression des résultats. Ecol. Medit. 14: 149-166.
KNUDSEN M. 1902. Berichte uber die konstanten Bestimmung zur Aufstellung der Hydrographischen Tabellen. Dansk Videns. Selek, Skrifter Natuwidensk. math. 6 , part $12, \mathbf{n}^{\circ} 1$.

MANZANO, M.; CUSTODIO, E. \& R. PONCELA 1991. Contribución de la hidrogeoquímica al conocimiento de la hidrodinámica de los acuíferos del área de Doñana. II Simposio del Agua en Andalucía: 475-486.

MINISTERIO DE AGRICULTURA. 1986. Métodos oficiales de análisis (T.III). Dirección General de Política Alimentaria. Ministerio de Agricultura, Madrid.

WILLIAMS, W.D. 1966. Conductivity and the concentration of total dissolved solids in Australian lakes. Aust. J. Mar. Freshwat. Res. 17: 169-176.

WILLIAMS, W.D. 1986. Conductivity and salinity in Australian salt lakes. Aust. J. Mar. Freshwat. Res. 37: 177 182.

ZAR, J.H. 1984. Biostatistical Analysis, Prentice Hall Inc. 